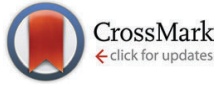

Cite this: Phys. Chem. Chem. Phys., $2015,17,16315$

\section{Adsorption of choline benzoate ionic liquid on graphene, silicene, germanene and boron-nitride nanosheets: a DFT perspective}

\author{
Gregorio García, ${ }^{a}$ Mert Atilhan ${ }^{\mathrm{b}}$ and Santiago Aparicio*a
}

\begin{abstract}
The adsorption of choline benzoate ([CH][BE]) ionic liquid (IL) on the surface of different hexagonal nanosheets has been studied using Density Functional Theory (DFT) methods. For this, the interaction mechanism, binding energies and electronic structure of $[\mathrm{CH}][\mathrm{BE}]$ ionic liquid on four types of nanosheets, i.e., graphene, silicene, germanene and boron-nitride, were estimated and compared. The adsorption of $[\mathrm{CH}][\mathrm{BE}]$ ionic liquid on different nanosheets is mainly featured by van der Waals forces, leading to strong benzoate ion-surface $\pi$-stacking. Likewise, there is also an important charge transfer from the anion to the sheet. The electronic structure analysis shows that Si- and Ge-based sheets lead to the largest changes in the HOMO and LUMO levels of choline benzoate. This paper provides new insights into the capability of DFT methods to provide useful information about the adsorption of ionic liquids on nanosheets and how ionic liquid features could be tuned through the adsorption on the suitable nanosheet.
\end{abstract}

Received 27th April 2015, Accepted 26th May 2015 DOI: $10.1039 /$ c5cp02432c

www.rsc.org/pccp because of its importance both for basic science and for applied purposes..$^{8-12}$

ILs are emerging as an attractive alternative to conventional organic solvents due to their special chemical and physical properties: negligible vapor pressures, high thermal and chemical stability, non-flammability or good solvent capacity for a wide range of organic, inorganic, polymeric and organometallic compounds, and the possibility of designing task-specific ILs through the suitable combination of cations and anions. ${ }^{13}$ Therefore, ILs have been considered for applications in different technological fields such as lubrication, solvent extraction, catalytic processes or electrochemical applications. ${ }^{14}$ In addition, the behavior of ILs with regard to graphene surfaces has also been studied, using both experimental and theoretical approaches, which have led to propose relevant applications in several technological fields, such as full cells, supercapacitors, solar cells or storage devices. ${ }^{8,10,12,15}$ As a matter of fact, both theoretical and experimental studies have been conducted to elucidate the mechanism of interaction between ILs and graphene. ${ }^{8,10,11,15-17}$ Nevertheless, most available studies are limited to a reduced number of classic types of ILs, such as imidazole based ionic liquids.

Beyond the graphene sheets, other hexagonal 2D nanosheets such as silicene, germanene and boron-nitride analogues have also attracted increasing attention. ${ }^{1,18}$ Silicene and germanene are the graphene counterparts based on silicon and germanium, which are also elements of group IV. These materials also possess a honeycomb structure similar to graphene, and thus, they also show the most outstanding properties of graphene such as high carrier mobility, ferromagnetism or zero band gap. ${ }^{1,18}$

\footnotetext{
${ }^{a}$ Department of Chemistry, University of Burgos, 09001 Burgos, Spain.

E-mail: sapar@ubu.es

${ }^{b}$ Department of Chemical Engineering, Qatar University, P.O. Box 2713,

Doha, Qatar
} 
Similarly, Si and Ge based nanosheets are expected to offer an alternative for the enhancement of the performance and scalability of the traditional silicon-based devices. ${ }^{1,19}$ Nonetheless, some remarkable differences should be noted between $\mathrm{C}$ and $\mathrm{Si} / \mathrm{Ge}$ based nanosheets. The buckled honeycomb structure of silicene and germanene leads to a significantly high chemical reactivity than graphene, and thus, to a much stronger trend for adsorption of atoms and molecules. ${ }^{1,19,20}$ In silicene/germanene sheets, silicon/germanium shows $\mathrm{sp}^{3}$ hybridization, which is the most favorable configuration in comparison with the $\mathrm{sp}^{2}$ or the mixed $\mathrm{sp}^{2}-\mathrm{sp}^{3}$ orbitals. This $\mathrm{sp}^{3}$ hybridization leads to common covalent $\mathrm{Si}-\mathrm{Si} / \mathrm{Ge}-\mathrm{Ge}$ bonds in a low buckled structure. ${ }^{1,19,21} 2 \mathrm{D}$ heterostructures, such as boron nitride (BN), are composed of an equal number of alternating boron and nitrogen atoms with $\mathrm{sp}^{2}$ hybridization in a honeycomb arrangement, with a similar structure to graphene. BN sheets are intrinsically insulators (or wide gap semiconductors), stable up to $1000 \mathrm{~K}$ and more resistant to oxidation than graphene. ${ }^{22}$ All these properties justify the great interest on $\mathrm{Si}$, Ge and $\mathrm{BN}$ nanosheets. As for graphene, properties of different $\mathrm{Si}, \mathrm{Ge}$ and $\mathrm{BN}$ sheets can be tuned through the adsorption of different molecules. This has led to several studies in the last few years dealing with the surface functionalization of $\mathrm{Si}^{1,18,19,23-25} \mathrm{Ge}^{1,19,20}$ or $\mathrm{BN}^{1,22,26-29}$ nanosheets. These studies point out that a new hybrid system based on hexagonal nanosheets with concrete features could be designed through the adsorption of adequate molecules on the surface. In spite of the wide interest on 2D surface functionalization through molecular adsorption, there is still scarce information about the adsorption of different molecules on silicene, germanene and $\mathrm{BN}$ sheets. Experimental techniques, such as atomic force microscopy, scanning tunneling microscopy or $\mathrm{X}$-ray spectroscopy, as well as theoretical methods like ab initio quantum chemistry methods (mainly Density Functional Theory, DFT), molecular dynamics (MD) and Monte Carlo (MC) simulations have been applied to study the ionic-liquid-graphene interface. ${ }^{8,10,11,15-17}$ As previously noted, those studies are mainly limited to classic ILs. Few studies on the adsorption of ILs on BN nanosheets have been also reported, ${ }^{29,30}$ while studies on the adsorption of IL on silicene or germanene surfaces have been not published.

Although DFT simulations are limited to relatively simple theoretical models, they have proven their ability in providing useful information about the design of functionalized nanosheets. ${ }^{6-8}$ In addition, DFT methods provide a deeper knowledge of the interaction of ILs at the surface, explaining their electronic structure and shedding light on the interaction mechanism. Therefore, a DFT study on the adsorption of ILs on $\mathrm{C}, \mathrm{Si}, \mathrm{Ge}$ and $\mathrm{BN}$ based hexagonal nanosheets is reported in this work. Concretely, the adsorption of choline benzoate ([CH] $]$ BE], Fig. 1) ionic liquid was studied here. Choline $([\mathrm{CH}])$ based ionic liquids are a new family of ionic liquids ${ }^{31}$ with suitable properties such as null toxicity, high biodegradability and low cost. Likewise, the combination of a choline cation with a benzoate anion ([BE]) leads to an ionic liquid composed completely of biomaterials, ${ }^{32}$ which can be produced at very low cost, ${ }^{33}$ and with null environmental impact. Thus, $[\mathrm{CH}][\mathrm{BE}]$ ionic liquid

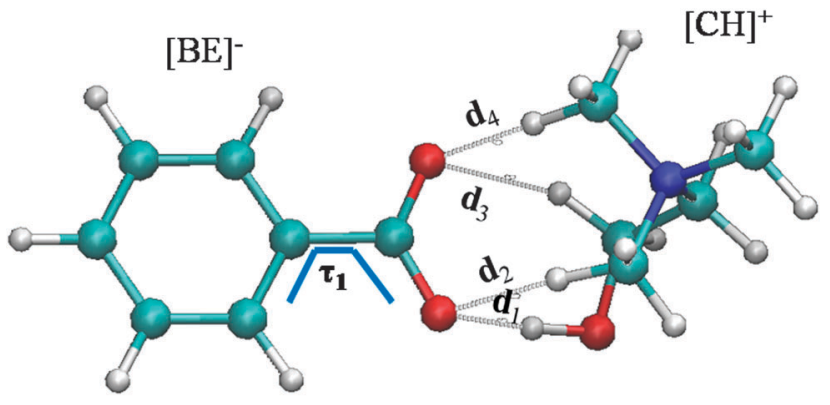

Fig. 1 Optimized structure at the PBE/DZP level for isolated choline benzoate ([CH][BE]) along the main structural parameters related to intermolecular interactions. Atom colour code: (green) carbon, (red) oxygen, (blue) nitrogen and (white) hydrogen.

has been selected in this work as a first example in the study of the adsorption of ILs on different nanosheets.

\section{Theoretical methodology}

DFT simulations were carried out using the generalized gradient approximation of Perdew, Burke and Ernzerhof $(\mathrm{PBE})^{34}$ as implemented in the SIESTA 3.2 package, ${ }^{35}$ along with norm-conserving Troullier-Martins pseudopotentials ${ }^{36}$ and numerical double- $\zeta$ polarized (DZP) basis sets. All calculations were done using an energy mesh cut-off of 400 Ry and a $k$-point mesh of $8 \times 8 \times 8$ in the Monkhorst-Pack scheme. ${ }^{37}$ Model nanosheets were described to have a $2 \mathrm{D}$ honeycomb structure of 128 atoms under periodic boundary conditions (PBC) applied in the three space directions. $\mathrm{PBC}$ in the direction normal to the nanosheet surface was $30 \AA$, long enough to avoid interaction between image layers. Structural relaxation was done by conjugate gradients, with convergence criteria that forces acting on all atoms do not exceed $0.03 \mathrm{eV}^{-1}$.

The PBE functional has been successfully applied to study interaction between different molecules and $\mathrm{C},{ }^{38,39} \mathrm{Si}^{24,40} \mathrm{Ge}^{41}$ or $\mathrm{BN}^{22,28}$ nanosheets. Long range dispersion interactions are expected to be important for an adequate description of ILnanosheet systems under study. In this sense, it is well known that the most common GGA (generalized gradient approximation) functionals have shortcomings of the adequate description of long range dispersion interactions. ${ }^{42}$ Some reported results have shown that dispersion corrections are not required in some cases for which molecules develop van der Waals interactions. ${ }^{7,39}$ On the other hand, many studies have pointed out the need for the adequate description of dispersive interactions. ${ }^{7,20,25,26,43}$ Therefore, dispersion corrections, according to Grimme's scheme, ${ }^{44}$ over the PBE functional (PBE-D2) were also employed to obtain information about the effect of dispersion corrections on calculated properties.

$[\mathrm{CH}][\mathrm{BE}]-$ nanosheet models were built by (randomly) placing the geometry optimized ionic liquid onto the nanosheet. In this starting geometry, the IL was placed to allow a $\pi$-stacking interaction between the phenyl motif and the surface with a distance of around $3.5 \AA$ (typical $\pi$-stacking distance). These starting geometries were optimized. Then, the relative disposition 
(through longitudinal displacements) over the surface as well as the rotational angle through the main bond between both ions (see $d_{1}$, Fig. 1) holding the above mentioned $\pi$-stacking between the phenyl motif and the surface, have been assessed. These analyses were carried out based on previously optimized geometries through single point calculations. From previous single points, choline benzoate ionic liquid was placed at the most stable disposition over each nanosheet and these $[\mathrm{CH}][\mathrm{BE}]-$ nanosheet models were optimized, which led to the optimized structures discussed here.

Binding energies, BEs, between $[\mathrm{CH}][\mathrm{BE}]$ and the corresponding nanosheets were estimated as

$$
\mathrm{BE}_{\mathrm{IL}-\mathrm{S}}=\left(E_{\mathrm{S}}+E_{\mathrm{IL}}\right)-E_{\mathrm{IL}-\mathrm{S}}
$$

where $E_{\mathrm{S}}, E_{\mathrm{IL}}$, and $E_{\mathrm{IL}-\mathrm{S}}$ stand for the total energy of the sheet, ionic pair and total energy of the IL-nanosheet, respectively. BE corresponding to the interaction between ions is defined as

$$
\mathrm{BE}_{\mathrm{IL}}=\left(E_{\text {cat }}+E_{\mathrm{ani}}\right)-E_{\mathrm{IL}}
$$

where $E_{\text {cat }}$ and $E_{\text {ani }}$ are the total energy of the cation and the anion, respectively. Charge transfer (CT) between graphene and IL has been studied through Hirshfeld ${ }^{45}$ and ChelpG $^{46}$ atomic charges. The ChelpG scheme has proven to be adequate for describing atomic charges in ILs, ${ }^{47,48}$ while the Hirshfeld model is an alternative definition of atomic charges based on partitioning of the electron density. ${ }^{45}$ Interactions between the IL and the nanosheets were featured through both a topological analysis of the electron density, according Bader's ${ }^{49}$ theory (Atoms in Molecules, AIM), and the analysis of the reduced density gradient (RGD) at low densities. ${ }^{50}$ According to AIM theory, ${ }^{49}$ there are four kinds of critical points, but because of the characteristics of the studied systems, and for improving and clarifying data analysis, attention has been paid to bond critical points (BCPs), which improves the criteria for considering the presence of intermolecular interactions, through the computed electronic density $(\rho)$ and its Laplacian $\left(\nabla^{2} \rho\right)$. Nevertheless, some information regarding the adsorption process could also be obtained from ring and cage critical points (RCP and CCP, respectively). RGD analysis is able to find non-covalent interactions based on the peaks that appear at low densities. Thus, the visualization of RGD iso-surfaces for these peaks allows the localization of weak interactions. The strength and nature of the interactions are determined through the sign of the second density Hessian eigenvalue. ${ }^{50}$ AIM and RGD analyses were carried out using the MultiWFN code. ${ }^{51}$ Partial density of states (PDOS) was extracted using the program developed by A. Postnikov. ${ }^{52}$

\section{Results and discussion}

\subsection{Choline benzoate on nanosheets}

Fig. 1 shows the optimized structure of the isolated $[\mathrm{CH}][\mathrm{BE}]$ ionic pair. As can be seen, there are several intermolecular interactions between both ions, labeled as $d_{1}-d_{4}$. All these interactions are hydrogen bonds, where oxygen atoms of the carboxyl group in the benzoate anion are hydrogen bond acceptors. As seen in Table 1,
Table 1 Main molecular parameters for the most stables structures optimized at PBE/DZP and PBE-D2/DZP levels for [CH][BE] ionic liquid. AIM parameters related with intermolecular interactions (electronic density, $\rho$, and its ILplacian, $\nabla^{2} \rho$ ) as well as the total charge over choline $\left(q^{+}\right)$ and benzoate $\left(q^{-}\right)$ions computed according to ChelpG/Hirshfeld schemes

\begin{tabular}{|c|c|c|c|c|c|c|}
\hline & \multicolumn{3}{|l|}{ PBE/DZP } & \multicolumn{3}{|c|}{ PBE-D2/DZP } \\
\hline & Length/A & $\rho /$ a.u. & $\nabla^{2} \rho /$ a.u. & Length/Å & $\rho /$ a.u. & $\nabla^{2} \rho /$ a.u. \\
\hline$d_{1}$ & 1.673 & 0.0668 & 0.1267 & 1.673 & 0.0668 & 0.1303 \\
\hline$d_{2}$ & 2.065 & 0.0221 & 0.0685 & 1.980 & 0.0265 & 0.0843 \\
\hline$d_{3}$ & 2.172 & 0.0193 & 0.0601 & 2.122 & 0.0213 & 0.0664 \\
\hline$d_{4}$ & 1.841 & 0.0356 & 0.1090 & 1.816 & 0.0382 & 0.1155 \\
\hline$\tau_{1} /{ }^{\circ}$ & & 0.0 & & & 0.0 & \\
\hline$q^{+} / e^{-a}$ & & $0.69 / 0.50$ & & & $0.68 / 0.48$ & \\
\hline
\end{tabular}
are also collected. See Fig. 1 for labeling

both intermolecular distances and AIM parameters (BCPs associated with these intermolecular interactions) are only slightly affected by the use of dispersive corrections (PBE-D2 functional). The intermolecular hydrogen bond between the hydroxyl group of the choline cation and the $\mathrm{COO}^{-}$group of the benzoate anion $\left(d_{1}\right)$ is the strongest one. This hydrogen bond yields a distance of $1.673 \AA$ and $\rho=0.0668$ a.u. (at PBE/DZP level), which are similar to those described previously for $[\mathrm{CH}][\mathrm{BE}]$ ionic liquid. ${ }^{48}$ Despite the presence of intermolecular hydrogen bonds, it is well known that cation-anion interactions in ionic liquids are mainly governed by Coulombic forces. In addition, there is a remarkable charge transfer of $0.69 / 0.50 e^{-}$(estimated through ChelpG/ Hirshfeld at the PBE/DZP level).

The most stable configurations of IL-nanosheet systems along with their calculated properties are reported in Fig. 2-5 and Tables 2-5. Following the labeling used in Fig. 1, intermolecular interactions between both ions are labeled as $d_{1}-d_{4}$. Regarding $[\mathrm{CH}][\mathrm{BE}]$ on top of the graphene sheet, intermolecular interactions $d_{1}-d_{4}$ are not dramatically affected during the adsorption process. For example, the average bond variation after the adsorption process is $0.026 \AA / 0.070 \AA$ using the PBE/PBE-D2 functional. These small bond variations point out that the main features of the ionic liquid are also present for the ionic liquid on top of the nanosheet. A similar trend was found for atomic charges, which are similar to those estimated in the absence of the graphene surface. At the PBE-D2/DZP level and by using the ChelpG scheme, a cation/anion loses its positive/negative charge $\left(0.07 / 0.13 e^{-}\right)$, while the graphene surface has a charge of $-0.06 e^{-}$. The negative sign of graphene charge indicates a small charge transfer from the ionic liquid (concretely from the anion) to the surface. The remaining anionic lost charge stands for the increase noted in the cationic charge. Thus the adsorption of IL on graphene leads to a charge transfer between ions of $0.34 e^{-}$according the ChelpG scheme at the PBE/DZP level. In general terms, similar conclusions are obtained for the interaction between both ions in presence of boron-nitride sheets. The Hirshfeld model predicts a higher charge transfer between the anion and the BN-based surface $\left(0.26 e^{-}\right)$.

The adsorption of choline benzoate on silicene leads to a proton transfer between both ions (Fig. 3). As can be seen in Table 3, 

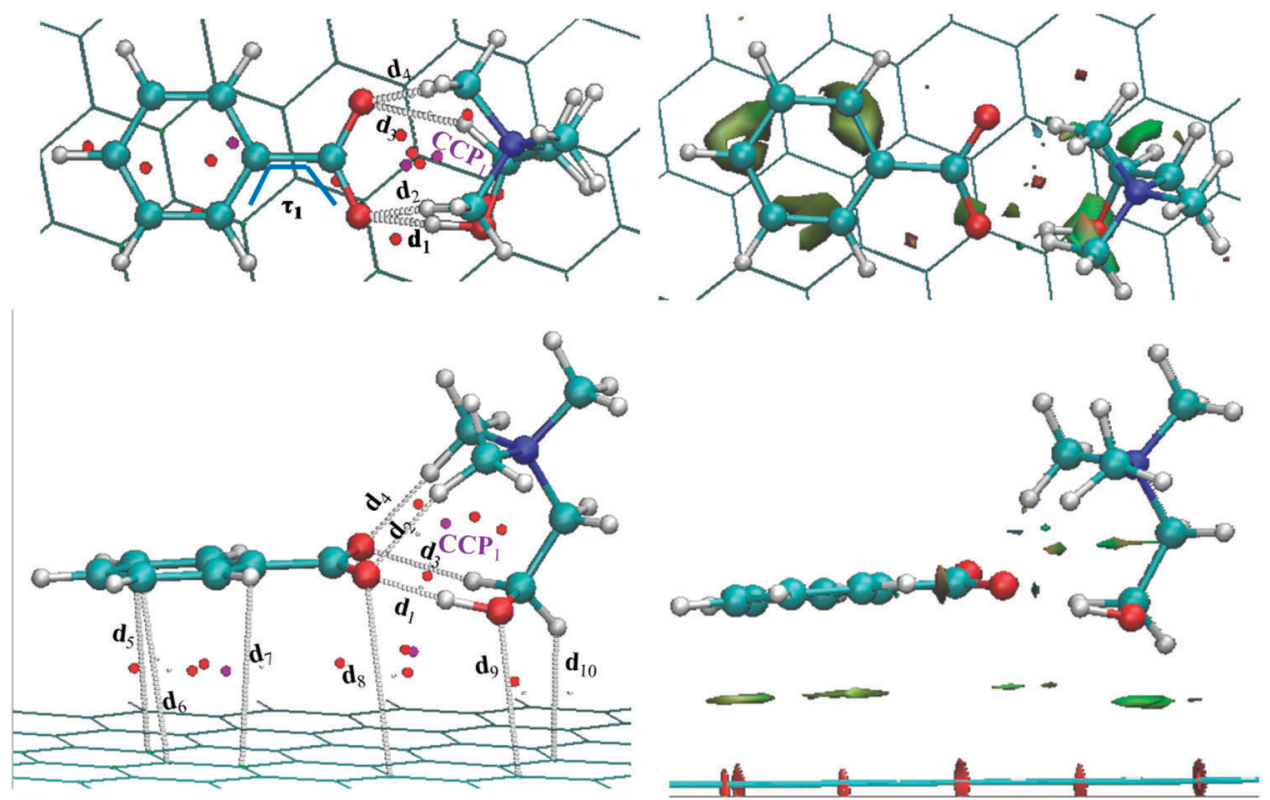

Fig. 2 Top (up) and side (bottom) view of the optimized structure at the PBE/DZP level corresponding to the [CH][BE]-graphene (II [CH][Be]-C) system along the structural parameters related to intermolecular interactions (left) and RGD iso-surfaces (right), whose green colour indicates van der Waals interactions. Atom colour code: (green) carbon, (red) oxygen, (blue) nitrogen and (white) hydrogen. Red and purple points stand for RCP and CCP, respectively, related to intermolecular interactions. BCPs were omitted for simplicity.
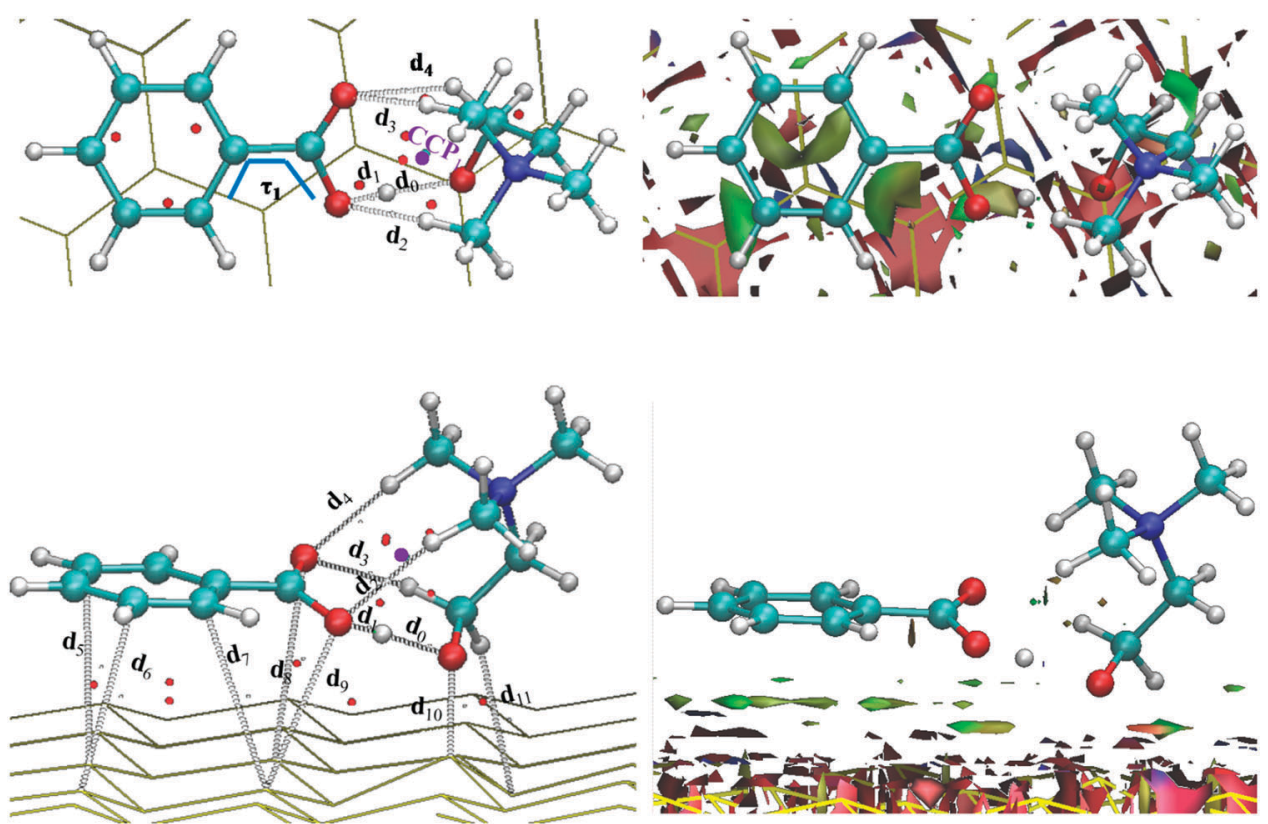

Fig. 3 Top (up) and side (bottom) view of the optimized structure at the PBE/DZP level corresponding to the [CH][BE]-silicene (III [CH][Be]-Si) system along the structural parameters related to intermolecular interactions (left) and RGD iso-surfaces (right), whose green colour indicates van der Waals interactions. Atom colour code: (green) carbon, (red) oxygen, (blue) nitrogen, (white) hydrogen and (yellow) silicon. Red and purple points stand for RCP and $\mathrm{CCP}$, respectively, related to intermolecular interactions. BCPs were omitted for simplicity.

$d_{1}=1.026 \AA$ at the PBE/DZP level, which is a typical O-H bond length, in agreement with its high electronic density value and negative Laplacian. Distance $d_{0}$ has been defined as the bond between both $\mathrm{O}$ and $\mathrm{H}$ atoms corresponding to the hydroxyl choline motif (Fig. 3). This distance $\left(d_{0}\right)$ yields features corresponding to an intermolecular hydrogen bond, such a $d_{1}$ for the isolated ILs. All these factors point out a clear proton transfer from the cation to the anion. The ChelpG/Hirshfeld model yields an interionic $\mathrm{CT}=0.37 e^{-} / 0.28 e^{-}$, whereas charge transfer from the anion to the silicene is $0.06 e^{-} / 0.54 e^{-}$. Hirshfeld atomic charges lead to a greater charge transfer up to the surface with the consequent negative charge reduction over the benzoate, 

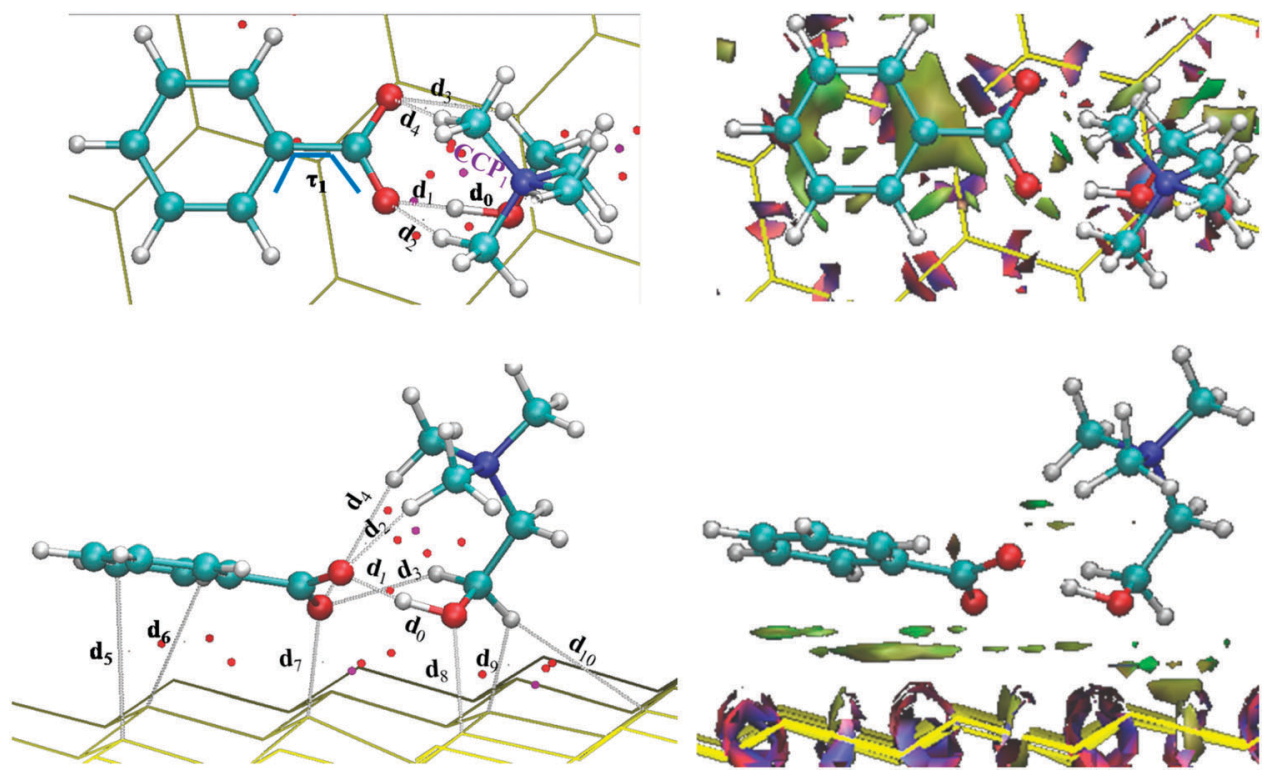

Fig. 4 Top (up) and side (bottom) view of the optimized structure at the PBE/DZP level corresponding to the [CH][BE]-germanene (IV [CH][Be]-Be) system along the structural parameters related to intermolecular interactions (left) and RGD iso-surfaces (right), whose green colour indicates van der Waals interactions. Atom colour code: (green) carbon, (red) oxygen, (blue) nitrogen, (white) hydrogen and (yellow) germanium. Red and purple points stand for RCP and CCP, respectively, related to intermolecular interactions. BCPs were omitted for simplicity.

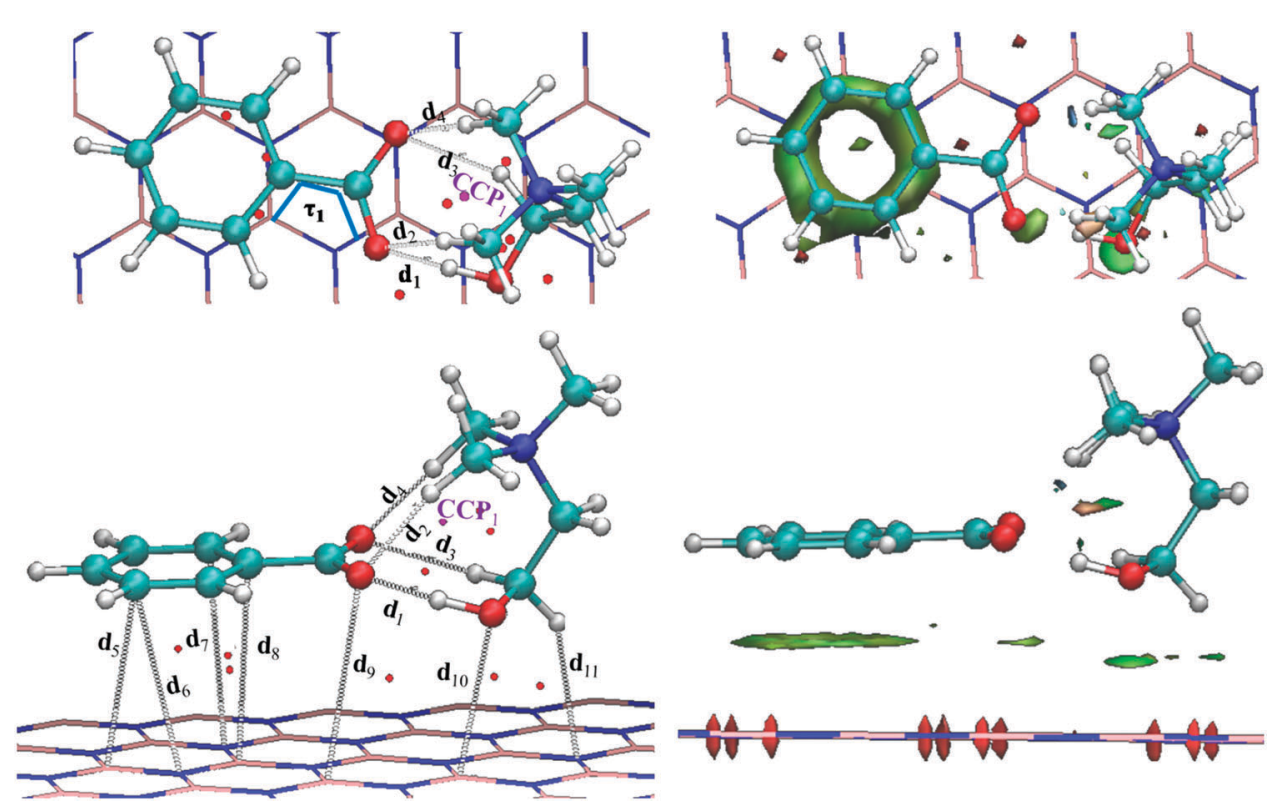

Fig. 5 Top (up) and side (bottom) view of the optimized structure at the PBE/DZP level corresponding to the [CH][BE]-boron-nitride (V [CH][Be]-BN) system along structural parameters related to intermolecular interactions (left) and RGD iso-surfaces (right), whose green colour indicates van der Waals interactions. Atom colour code: (green) carbon, (red) oxygen, (blue) nitrogen, (white) hydrogen and (pink) boron. Red and purple points stand for RCP and $\mathrm{CCP}$, respectively, related with intermolecular interactions. BCPs were omitted for simplicity.

while there is a decrease in the inter-ionic CT. The PBE-D2 functional also predicts a proton transfer between both ions. However, there is a strengthening of $d_{0}$ interaction (which decreases/increase its length/electronic density) in comparison with the optimized structure at the PBE level. Both ChelpG and Hirshfeld models yield smaller inter-ionic CT (of around $0.30 e^{-}$) than isolated $\mathrm{IL}$, while $\mathrm{CT}$ to the silicene surface increases up to $0.65 e^{-}$.
The optimized structure of $[\mathrm{CH}][\mathrm{BE}]$ on the Ge based surface at the PBE/DZP level is shown in Fig. 4. As can be seen in Table $4, d_{1}$ suffers a shortening $(0.261 \AA)$ with an increase in electronic density due to the adsorption process, which results in the strengthening of the interaction between both ions. In addition, the relative disposition of choline hinders the interaction $d_{4}$ (the corresponding the BCP was not found). According to the Hirshfeld model, cation/anion charge becomes more 
Table 2 Main molecular parameters for the most stables structures optimized at PBE/DZP and PBE-D2/DZP levels. For the [CH][BE]-graphene system, AIM parameters related with intermolecular interactions (electronic density, $\rho$, and its laplacian, $\nabla^{2} \rho$ ) as well as the total charge over choline $\left(q^{+}\right)$, benzoate $\left(q^{-}\right)$ions and sheet $\left(q^{s}\right)$ computed according to ChelpG/ Hirshfeld schemes are also collected. See Fig. 2 for labeling

\begin{tabular}{|c|c|c|c|c|c|c|}
\hline & \multicolumn{3}{|l|}{ PBE/DZP } & \multicolumn{3}{|c|}{ PBE-D2/DZP } \\
\hline & Length/Å & $\rho /$ a.u. & $\nabla^{2} \rho /$ a.u. & Length/Å & $\rho /$ a.u. & $\nabla^{2} \rho /$ a.u. \\
\hline$d_{1}$ & 1.653 & 0.0702 & 0.1245 & 1.618 & 0.0772 & 0.1177 \\
\hline$d_{2}$ & 2.048 & 0.0230 & 0.0718 & 2.059 & 0.0222 & 0.0711 \\
\hline$d_{3}$ & 2.185 & 0.0188 & 0.0585 & 2.183 & 0.0191 & 0.0595 \\
\hline$d_{4}$ & 1.895 & 0.0314 & 0.0977 & 1.901 & 0.0309 & 0.0972 \\
\hline$\tau_{1} /^{\circ}$ & 0.0 & & & 0.0 & & \\
\hline$d_{\mathrm{eq}} / \AA$ & 3.651 & & & 3.161 & & \\
\hline$d_{5}$ & 3.601 & 0.0039 & 0.0119 & 3.126 & 0.0086 & 0.0275 \\
\hline$d_{6}$ & 3.507 & 0.0044 & 0.0135 & 3.198 & 0.0080 & 0.0271 \\
\hline$d_{7}$ & 3.768 & 0.0030 & 0.0092 & 3.240 & 0.0075 & 0.0256 \\
\hline$d_{8}$ & 3.749 & 0.0022 & 0.0071 & 3.305 & 0.0065 & 0.0239 \\
\hline$d_{9}$ & 3.154 & 0.0072 & 0.0251 & 2.930 & 0.0053 & 0.0194 \\
\hline$d_{10}$ & 2.762 & 0.0182 & 0.0354 & 2.485 & 0.0115 & 0.0446 \\
\hline$\sum(\mathrm{BCP})^{a}$ & & 0.0389 & 0.1022 & & 0.0474 & 0.1861 \\
\hline$\sum(\mathrm{RCP})^{b}$ & & 0.0521 & 0.2316 & & 0.0607 & 0.2142 \\
\hline$\overline{\mathrm{CCP} 1}$ & & 0.0037 & 0.0175 & & 0.0603 & 0.2342 \\
\hline$\sum(\mathrm{CCP})^{c}$ & & 0.0032 & 0.0111 & & 0.0040 & 0.0187 \\
\hline$q^{+} / e^{-}$ & $0.66 / 0.50$ & & & $0.61 / 0.50$ & & \\
\hline$q^{-} / e^{-}$ & $-0.65 /-0.48$ & & & $-0.55 /-0.47$ & & \\
\hline$q^{\mathrm{s}} / e^{-}$ & $-0.01 /-0.02$ & & & $-0.06 /-0.03$ & & \\
\hline$\Delta d_{\mathrm{C}-\mathrm{C}}{ }^{d} / \AA$ & 0.009 & & & 0.009 & & \\
\hline
\end{tabular}

${ }^{a} \sum(\mathrm{BCP}) / \sum(\mathrm{RCP}) / \sum(\mathrm{CCP})$ represents the sum of $\rho$ or $\nabla^{2} \rho$ for those $\mathrm{BCP} / \mathrm{RCP} / \mathrm{CCP}$ related to intermolecular interactions between the IL and the sheet. ${ }^{b} \sum(\mathrm{BCP}) / \sum(\mathrm{RCP}) / \sum(\mathrm{CCP})$ represents the sum of $\rho$ or $\nabla^{2} \rho$ for those $\mathrm{BCP} / \mathrm{RCP} / \mathrm{CCP}$ related to intermolecular interactions between the IL and the sheet. ${ }^{c} \sum(\mathrm{BCP}) / \sum(\mathrm{RCP}) / \sum(\mathrm{CCP})$ represents the sum of $\rho$ or $\nabla^{2} \rho$ for those $\mathrm{BCP} / \mathrm{RCP} / \mathrm{CCP}$ related to intermolecular interactions between the IL and the sheet. ${ }^{d}$ Difference value of $\mathrm{C}-\mathrm{C}$ bonds corresponding to nanosheets after and before of the adsorption process.

positive/less negative, whereby interionic CT decreases $0.19 e^{-}$, while there is an important CT from the anion up to the germanene $\left(\mathrm{CT}=0.54 e^{-}\right)$. The use of dispersion corrections in the PBE functional (PBE-D2) leads to a proton charge transfer from the cation to the anions $\left(d_{0}\right.$ and $d_{1}$ bond lengths and AIM parameters computed at the PBE-D2/DZP level in Table 4).

In short, the most dramatic changes during the adsorption process are the proton transfer in the IL-Si system (also in IL-Ge at the PBE-D2/DZP level), and CT computed according to the Hirshfeld model. Isolated choline benzoate, IL-graphene and IL-BN systems yield an inter-ionic CT of $\sim 0.50 e^{-}$, while this CT is equal to $0.28 e^{-} / 0.19 e^{-}$for the IL on the silicene/ germanene surface (at the PBE/DZP level). In addition, due to the adsorption process, the new arrangement between both ions leads to a new cage critical point (labeled as CCP1, Fig. 2-5 and Tables 2-5), whose $\rho$ could provide some information on interionic interaction strength. This CCP yields similar $\rho$ values for $[\mathrm{CH}][\mathrm{BE}]$ on top of $\mathrm{C}, \mathrm{Si}$ and $\mathrm{BN}$ sheets, while the largest $\rho$ values are obtained for IL on top of germanene.

\subsection{Adsorption mechanism: $[\mathrm{CH}][\mathrm{BE}]-$ nanosheet interactions}

As concerns the changes in the structure of studied nanosheets during the adsorption process, the $\Delta d_{\mathrm{X}-\mathrm{x}}$ parameter (see Tables 2-5) has been defined as the difference value of $\mathrm{X}-\mathrm{X}$ after and before the adsorption process ( $\mathrm{X}-\mathrm{X}=\mathrm{C}-\mathrm{C}, \mathrm{Si}-\mathrm{Si}, \mathrm{Ge}-\mathrm{Ge}$ or $\mathrm{B}-\mathrm{N}$ bonds).
Table 3 Main molecular parameters for the most stables structures optimized at PBE/DZP and PBE-D2/DZP levels. For the [CH][BE]-silicene system, AIM parameters related to intermolecular interactions (electronic density, $\rho$, and its Laplacian, $\nabla^{2} \rho$ ) as well as the total charge over choline $\left(q^{+}\right)$, benzoate $\left(q^{-}\right)$ions and sheet $\left(q^{\text {s}}\right)$ computed according to ChelpG/ Hirshfeld schemes are also collected. See Fig. 3 for labeling

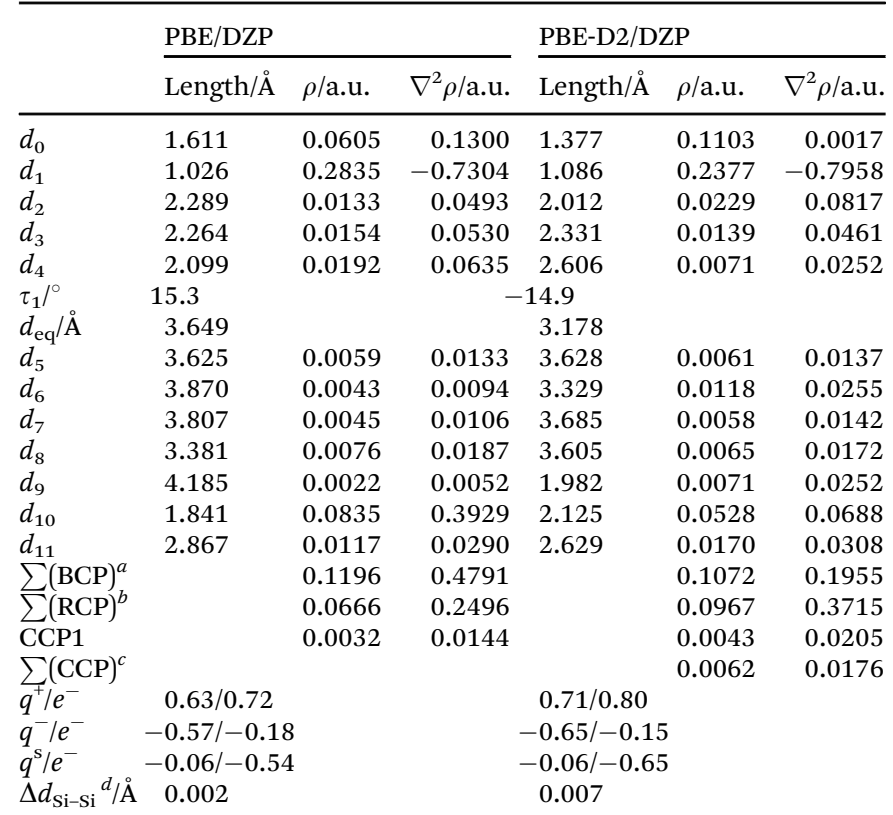

${ }^{a} \sum(\mathrm{BCP}) / \sum(\mathrm{RCP}) / \sum(\mathrm{CCP})$ represents the sum of $\rho$ or $\nabla^{2} \rho$ for those $\mathrm{BCP} / \mathrm{RCP} / \mathrm{CCP}$ related to intermolecular interactions between the IL and the sheet. ${ }^{b} \sum(\mathrm{BCP}) / \sum(\mathrm{RCP}) / \sum(\mathrm{CCP})$ represents the sum of $\rho$ or $\nabla^{2} \rho$ for those BCP/RCP/CCP related to intermolecular interactions between the IL and the sheet. ${ }^{c} \sum(\mathrm{BCP}) / \sum(\mathrm{RCP}) / \sum$ (CCP) represents the sum of $\rho$ or $\nabla^{2} \rho$ for those BCP/RCP/CCP related to intermolecular interactions between the IL and the sheet. ${ }^{d}$ Difference value of $\mathrm{Si}-\mathrm{Si}$ bonds corresponding to nanosheets after and before the adsorption process.

The studied IL-nanosheet systems and both theoretical levels (without dispersion corrections or dispersion corrected functional), $\Delta d_{\mathrm{x}-\mathrm{x}}<0.009 \AA$, point out that the nanosheet structure does not suffer important change upon the ionic liquid adsorption. In addition, graphene and boron-nitride sheets retain their planarity after the adsorption process, while silicene and germanene sheets also maintain their buckled structure.

Results reported in Fig. 2-5 show that the phenyl (benzoate) motif adopts a configuration parallel to the surface. The average interplanar distances between the phenyl moiety and different sheets (labeled as $d_{\text {eq }}$, Tables 2-5) were found to be of roughly $3.65 \AA$ for IL-graphene and IL-silicene systems and $\sim 3.74 \AA$ for IL-germanene and IL-boron nitride at the PBE/DZP level. For $[\mathrm{CH}][\mathrm{BE}]$ on the graphene sheet, $d_{\mathrm{eq}}$ computed at the PBE/DZP level lies close to the typical $\pi$-stacking distance (3.5-4.0 $\AA$ ). However, the PBE-D2/DZP level brings an approach $(\sim 0.50 \AA)$ between both planes.

The adsorption of the phenyl motif on graphene sets up three interactions with the surface $\left(d_{5}-d_{7}\right)$, whose average distance is $3.625 \AA$ (at PBE/DZP level). Besides, the carboxylate group also develops a link with the surface $\left(d_{8}\right)$. The dihedral angle between phenyl and carboxylate motifs $\left(\tau_{1}\right)$ is 0.0 degrees, which hinders the interaction between the carboxylate group 
Table 4 Main molecular parameters for the most stables structures optimized at PBE/DZP and PBE-D2/DZP levels. For the [CH][BE]-germanene system, AIM parameters related to intermolecular interactions (electronic density, $\rho$, and its Laplacian, $\left.\nabla^{2} \rho\right)$ as well as the total charge over choline $\left(q^{+}\right)$, benzoate $\left(q^{-}\right)$ions and the sheet $\left(q^{s}\right)$ computed according to Hirshfeld schemes are also collected. See Fig. 4 for labeling

\begin{tabular}{|c|c|c|c|c|c|c|}
\hline & \multicolumn{3}{|l|}{ PBE/DZP } & \multicolumn{3}{|c|}{$\underline{\text { PBE-D2/DZP }}$} \\
\hline & Length/Å & $\rho /$ a.u. & $\nabla^{2} \rho /$ a.u. & Length/Å & $\rho /$ a.u. & $\nabla^{2} \rho /$ a.u. \\
\hline$d_{0}$ & 1.079 & 0.2424 & -0.8545 & 1.404 & 0.1023 & 0.0424 \\
\hline$d_{1}$ & 1.412 & 0.1015 & 0.0335 & 1.077 & 0.2433 & -0.8680 \\
\hline$d_{2}$ & 2.070 & 0.0106 & 0.0346 & 2.309 & 0.0126 & 0.0479 \\
\hline$d_{3}$ & 2.469 & 0.0065 & 0.0151 & 2.287 & 0.0151 & 0.0523 \\
\hline$d_{4}$ & 3.071 & & & 2.064 & 0.0212 & 0.0683 \\
\hline$\tau_{1} /^{\circ}$ & -16.7 & & & 5.9 & & \\
\hline$d_{\mathrm{eq}} / \AA$ & 3.755 & & & 3.179 & & \\
\hline$d_{5}$ & 3.617 & 0.0023 & 0.0061 & 3.310 & 0.0102 & 0.0240 \\
\hline$d_{6}$ & 3.747 & & & 3.039 & 0.0076 & 0.0228 \\
\hline$d_{7}$ & 2.318 & 0.0428 & 0.1160 & 3.218 & 0.0100 & 0.0257 \\
\hline$d_{8}$ & 2.409 & 0.0385 & 0.0992 & 2.045 & 0.0789 & 0.1860 \\
\hline$d_{9}$ & 3.032 & 0.0078 & 0.0181 & 2.740 & 0.0127 & 0.0273 \\
\hline$d_{10}$ & 3.356 & 0.0046 & 0.0113 & 2.935 & 0.0094 & 0.0211 \\
\hline$\sum(\mathrm{BCP})^{a}$ & & 0.0959 & 0.2507 & & 0.1288 & 0.3070 \\
\hline$\sum(\mathrm{RCP})^{b}$ & & 0.1177 & 0.2249 & & 0.1024 & 0.3646 \\
\hline CCP1 & & 0.0038 & 0.0188 & & 0.0647 & 0.0156 \\
\hline$\sum(\mathrm{CCP})^{c}$ & & 0.0056 & 0.0150 & & 0.0087 & 0.0259 \\
\hline$q^{+} d / e^{-}$ & 0.81 & & & 0.75 & & \\
\hline$q^{-d} / e^{-}$ & -0.13 & & & -0.01 & & \\
\hline$q^{\mathrm{s} d} e^{-}$ & -0.68 & & & -0.75 & & \\
\hline$\Delta d_{\mathrm{Ge}-\mathrm{Ge}}{ }^{e} / \bar{f}$ & 0.001 & & & -0.009 & & \\
\hline
\end{tabular}

${ }^{a} \sum(\mathrm{BCP}) / \sum(\mathrm{RCP}) / \sum(\mathrm{CCP})$ represents the sum of $\rho$ or $\nabla^{2} \rho$ for those $\mathrm{BCP} / \mathrm{RCP} / \mathrm{CCP}$ related to intermolecular interactions between the IL and the sheet. ${ }^{b} \sum(\mathrm{BCP}) / \sum(\mathrm{RCP}) / \sum(\mathrm{CCP})$ represents the sum of $\rho$ or $\nabla^{2} \rho$ for those $\mathrm{BCP} / \mathrm{RCP} / \mathrm{CCP}$ related to intermolecular interactions between the IL and the sheet. ${ }^{c} \sum(\mathrm{BCP}) / \sum(\mathrm{RCP}) / \sum(\mathrm{CCP})$ represents the sum of $\rho$ or $\nabla^{2} \rho$ for those $\mathrm{BCP} / \mathrm{RCP} / \mathrm{CCP}$ related to intermolecular interactions between the IL and the sheet. ${ }^{d}$ ChelpG atomic charges were not computed since Breneman radii (which is needed to compute ChelpG charges) for the Ge atom is not available in the literature. ${ }^{e}$ Difference value of Ge-Ge bonds corresponding to nanosheets after and before the adsorption process.

(which keeps most of the negative charge) and the surface. Hence, the CT $\left(0.02 e^{-}\right.$according to the Hirshfeld scheme at the $\mathrm{PBE} / \mathrm{DZP}$ level) from the anion up to graphene sheets is close to zero. Silicene/germanene surfaces establish $3 / 2$ intermolecular bonds with the phenyl motifs (labeled as $d_{5}-d_{7} / d_{5}, d_{6}$ ), whose distances are larger than those found for the IL-graphene system (Fig. 3, 4 and Tables 3,4). Nonetheless, all of them yield larger electronic density values, which would point out to stronger interactions between the phenyl motif and the surface. Due to larger diameters of those rings forming the $\mathrm{Si}-\mathrm{Ge}$ surface, bonds between the surface and the phenyl are not perpendicular to the surface plane. In this case, $\tau_{1}=15.3$ degrees $/ 16.7$ degrees, which allows a shortening of the distance between the carboxylate group and the surface. Two/one interactions were found between the carboxylate group and the $\mathrm{Si} / \mathrm{Ge}$ sheet $\left(d_{8}, d_{9} / d_{7}\right)$. The CT $\left(0.54 e^{-} / 0.68 e^{-}\right.$according to the Hirshfeld scheme at the PBE/DZP level) between the anion and the germanene surface agrees with the shortest distance between the carboxyl group and the surface (2.318 ̊ at the PBE/DZP level). Likewise, the benzoate anion is able to develop five interactions with the boron-nitride surface (Fig. 5), four of them through the phenyl motif $\left(d_{5}-d_{8}\right)$ and the other one through the $\mathrm{COO}^{-}$group $\left(d_{9}\right)$. For $[\mathrm{CH}][\mathrm{BE}]$ on
Table 5 Main molecular parameters for the most stables structures optimized at PBE/DZP and PBE-D2/DZP levels. For the [CH][BE]-boronnitride system, AIM parameters related to intermolecular interactions (electronic density, $\rho$, and its laplacian, $\nabla^{2} \rho$ ) as well as total charge over choline $\left(q^{+}\right)$, benzoate $\left(q^{-}\right)$ions and sheet $\left(q^{s}\right)$ computed according to ChelpG/Hirshfeld schemes are also collected. See Fig. 5 for labeling

\begin{tabular}{|c|c|c|c|c|c|c|}
\hline & \multicolumn{3}{|l|}{ PBE/DZP } & \multicolumn{3}{|c|}{ PBE-D2/DZP } \\
\hline & Length/Å & $\rho /$ a.u. & $\nabla^{2} \rho /$ a.u. & Length/Å & $\rho /$ a.u. & $\nabla^{2} \rho /$ a.u. \\
\hline$d_{1}$ & 1.631 & 0.0743 & 0.1214 & 1.605 & 0.0803 & 0.1103 \\
\hline$d_{2}$ & 2.041 & 0.0233 & 0.0727 & 2.023 & 0.0240 & 0.0771 \\
\hline$d_{3}$ & 2.245 & 0.0167 & 0.0518 & 2.190 & 0.0189 & 0.0594 \\
\hline$d_{4}$ & 1.897 & 0.0314 & 0.0972 & 1.934 & 0.0287 & 0.0903 \\
\hline$\tau_{1} /^{\circ}$ & 1.7 & & & 3.8 & & \\
\hline$d_{\mathrm{eq}} / \AA ̊ ̊$ & 3.728 & & & 3.210 & & \\
\hline$d_{5}$ & 3.763 & 0.0030 & 0.0089 & 3.288 & 0.0079 & 0.0270 \\
\hline$d_{6}$ & 3.760 & 0.0031 & 0.0096 & 3.171 & 0.0075 & 0.0231 \\
\hline$d_{7}$ & 3.876 & 0.0023 & 0.0067 & 3.168 & 0.0076 & 0.0243 \\
\hline$d_{8}$ & 3.800 & 0.0025 & 0.0074 & 3.254 & 0.0077 & 0.0237 \\
\hline$d_{9}$ & 3.900 & 0.0017 & 0.0057 & 2.992 & 0.0084 & 0.0242 \\
\hline$d_{10}$ & 3.152 & 0.0068 & 0.0194 & 2.595 & 0.0183 & 0.0497 \\
\hline$d_{11}$ & 2.666 & 0.0082 & 0.0278 & 2.542 & 0.0112 & 0.0379 \\
\hline$\sum(\mathrm{BCP})^{a}$ & & 0.0276 & 0.0855 & & 0.0686 & 0.2099 \\
\hline$\sum(\mathrm{RCP})^{b}$ & & 0.0411 & 0.1864 & & 0.0999 & 0.4232 \\
\hline CCP1 & & 0.0038 & 0.0179 & & 0.0040 & 0.0189 \\
\hline$\sum(\mathrm{CCP})^{c}$ & & & & & 0.0072 & 0.0289 \\
\hline$q^{+} / e^{-}$ & $0.69 / 0.54$ & & & $0.64 / 0.59$ & & \\
\hline$q^{-} / e^{-}$ & $-0.66 /-0.43$ & & & $-0.57 /-0.33$ & & \\
\hline$q^{\mathrm{s}} / e^{-}$ & $-0.03 /-0.11$ & & & $-0.07 /-0.26$ & & \\
\hline$\Delta d_{\mathrm{B}-\mathrm{N}}{ }^{d} / \AA$ & 0.000 & & & 0.000 & & \\
\hline
\end{tabular}

${ }^{a} \sum(\mathrm{BCP}) / \sum(\mathrm{RCP}) / \sum(\mathrm{CCP})$ represents the sum of $\rho$ or $\nabla^{2} \rho$ for those $\mathrm{BCP} / \mathrm{RCP} / \mathrm{CCP}$ related with intermolecular interactions between the IL and the sheet. ${ }^{b} \sum(\mathrm{BCP}) / \sum(\mathrm{RCP}) / \sum(\mathrm{CCP})$ represents the sum of $\rho$ or $\nabla^{2} \rho$ for those $\mathrm{BCP} / \mathrm{RCP} / \mathrm{CCP}$ related with intermolecular interactions between the IL and the sheet. ${ }^{c} \sum(\mathrm{BCP}) / \sum(\mathrm{RCP}) / \sum(\mathrm{CCP})$ represents the sum of $\rho$ or $\nabla^{2} \rho$ for those BCP/RCP/CCP related with intermolecular interactions between the IL and the sheet. ${ }^{d}$ Difference value of B-N bonds corresponding to nanosheets after and before the adsorption process.

top of the BN sheet, $\tau_{1}$ is close to the planarity. This dihedral angle obstructs an approach $\left(d_{9}=3.900 \AA\right.$ at PBE/DZP level) between the $\mathrm{COO}^{-}$group of benzoate and the BN surface, leading to small CT from the anion to the BN sheet $\left(0.11 e^{-}\right.$according to the Hirshfeld scheme at the PBE/DZP level).

$[\mathrm{CH}]$-surface interactions are always carried out through two intermolecular bonds between the oxygen atom and the methylene group on the surface. Moreover, the germanene sheet allows another additional interaction with the methylene group (see Fig. 4). The shortest distance between $[\mathrm{CH}]$ and the surface is found for the IL-silicene system $\left(d_{10}=1.841 \AA\right.$ at the PBE/DZP level, Table 3). The presence of choline benzoate on the silicene surface leads to a deformation of the silicene sheet, which allows a shorter length for $d_{10}\left(d_{10}=1.841 \AA\right.$ for the IL-Si system at PBE/DZP). A different behavior is found with regard to the interaction strength (based on the distance and electronic density values) between the oxygen atom and the methylene group on the surface $\left(d_{10}=2.762 \AA / d_{11}=2.867 \AA / d_{9}=3.032 \AA\right.$ and $d_{10}=3.056 \AA / d_{11}=2.66 \AA$ for $\mathrm{IL}-\mathrm{C} / \mathrm{IL}-\mathrm{Si} / \mathrm{IL}-\mathrm{Ge} / \mathrm{IL}-\mathrm{BN}$ systems at PBE/DZP).

In addition to the information related with intermolecular IL-sheet interactions (labeled as $d_{5}-d_{11}$ ) and the AIM properties for their corresponding BCPs, main AIM parameters for RCPs 
and CCPs (red and purple points in Fig. 2-5) have also been collected. With the aim of looking for a relationship between the interaction strength for IL-nanosheet systems and AIM parameters, the total value of electronic density of the different calculated critical points is also gathered in Tables 2-5. Some relationships were found between AIM parameters for intermolecular interaction and binding energies. For example, Si and Ge based surfaces provide the largest value for the total electronic density for the BCPs related to the interactions between the ionic liquid and the surface $\left(\sum(\mathrm{BCP})\right)$. Silicene and germanene sheets allow stronger interactions than graphene or boron-nitride surfaces.

As seen for $d_{\text {eq }}$, the PBE-D2 functional estimates, in general, shorter intermolecular distances between the IL and the surfaces, which leads to an increase of some factors related with the intermolecular interactions, such as interaction strength (based on electronic density value) and IL-surface charge transfer.

The nature of the interaction between $[\mathrm{CH}][\mathrm{BE}]$ ionic liquid and different nanosheets was elucidated based on RGD isosurfaces. For $[\mathrm{CH}][\mathrm{BE}]-$ nanosheet systems, the RGD iso-surfaces are displayed in Fig. 2-5. The green color for the regions between the IL and the nanosheets points out that van der Waals interactions are the main forces responsible for the IL adsorption on nanosheets regardless of the considered nanosheet. The largest iso-surface between the phenyl motif and the BN surface is in concordance with the parallel disposition between the phenyl motif and the BN sheet. In short, anion-surface interactions should mainly contribute to the IL-surface binding energies.

As mentioned in previous sections, the available literature dealing with the adsorption of IL on nanosheets is very scarce (mainly limited to imidazole based ILs on graphene). Recently, Herrera et al. previously studied the adsorption of imidazole cation on the amino acid based anion graphene surface, ${ }^{8}$ while Shakourian-Fard et al. analyzed IL adsorption on BN nanosheets for classical ILs, ${ }^{29}$ both of them through DFT methods. The first employed functional was PBE-D2/DZP, while a meta-GGA functional was selected later. Both approximations should provide a good description for the dispersion forces. ${ }^{44,53}$ However, despite the IL (choline benzoate, aminoacid based or classical ILs), the surface (graphene or boron-nitride) or the applied methods (DFT-D2 or meta-GGA), similar structural features were inferred, i.e., the IL-surface distance of around 3.0 ̊ was found; IL-surface interactions were mainly featured by $\pi$-stacking interactions between aromatic ions (the imidazole cation for previously reported studies or the benzoate anion in this paper) and the surface.

\subsection{Binding energies}

Main intermolecular interactions between both ions as well as between the ionic liquid and the nanosheets were analyzed in previous sections. In addition, binding energies can be used as a measurement of the interaction strength. The binding energy of the interaction between both ions (BE $\mathrm{IL}_{\mathrm{IL}}$, Fig. 6) was $84.31 \mathrm{kcal} \mathrm{mol}^{-1}$ at the PBE/DZP level. This high value is mainly due to the important contribution from the Coulombic forces between both ions. Fig. 6 also shows $\mathrm{BE}_{\mathrm{IL}}$ of the $\mathrm{IL}$ on top of the nanosheets, which was

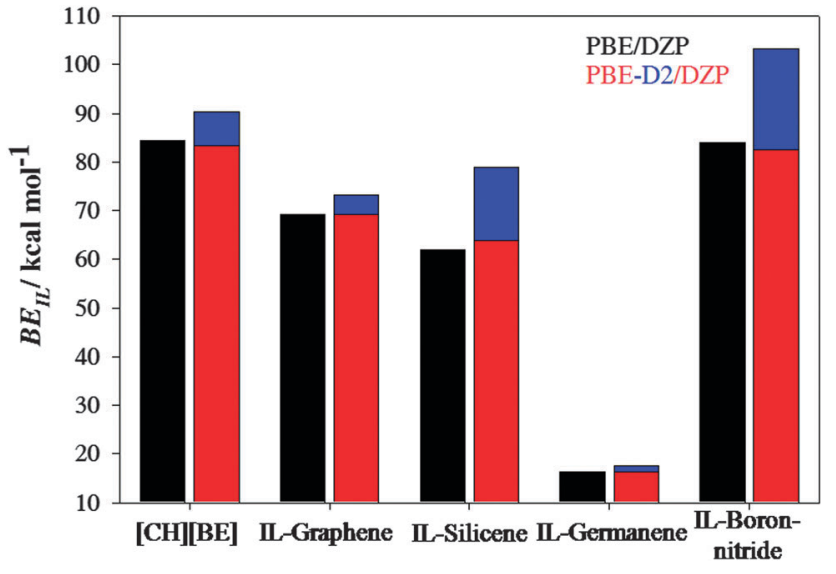

Fig. 6 Binding energies for the interaction between the both ions $\left(B E_{I L}\right)$ at PBE/DZP (black) and PBE-D2/DZP (red + blue) theoretical levels, wherein blue contributions stand for $\mathrm{BE}_{\mathrm{dis}}$.

calculated from the previously optimized IL geometry on top of each nanosheets. As seen in Fig. 6, $\mathrm{BE}_{\mathrm{IL}}$ decreases from IL on top of graphene up to IL on top of germanene, while $[\mathrm{CH}][\mathrm{BE}]$ on top of $\mathrm{BN}$ yields higher $\mathrm{BE}_{\mathrm{IL}}$. IL adsorption on graphene, silicene, or germanene leads to a $\mathrm{BE}_{\mathrm{IL}}$ of up to $16.34 \mathrm{kcal} \mathrm{mol}^{-1}$, while choline benzoate on top of boron-nitride yields a $\mathrm{BE}_{\mathrm{IL}}$ of $83.94 \mathrm{kcal} \mathrm{mol}^{-1}$. Hence, the adsorption of choline benzoate on the studied homo-nanosheets has important drawbacks on $\mathrm{BE}_{\mathrm{IL}}$. Albeit there is an important proton transfer from choline up to benzoate for IL-silicene systems; this fact would not have important effects on the interaction between both ions on the silicene surface. $\mathrm{BE}_{\mathrm{IL}}$ at the PBE-D2/DZP level (which is somewhat higher, mainly due to smaller intermolecular distances) follows a similar pattern during the adsorption process. Coulombic forces between ions play an important role in the interionic interactions. In fact, $[\mathrm{CH}][\mathrm{BE}]$ on silicene and germanene provides the lowest interionic $\mathrm{CT}$ and the lowest $\mathrm{BE}_{\mathrm{IL}}$. As said, intermolecular interactions (such as hydrogen bonds) are also an important contribution to $\mathrm{BE}_{\mathrm{IL}}$. In this sense, Fig. 6 also displays dispersion energy contribution $\left(\mathrm{BE}_{\mathrm{dis}, \mathrm{IL}}\right)$ to the total binding energy (quantified according to Grimme's approach ${ }^{44}$ for the PBE-D2 functional). $\mathrm{BE}_{\mathrm{dis}, \mathrm{IL}}$, which is very close to the difference between the binding energy between both (dispersion) corrected and non-corrected functionals, only provides a small contribution to the total $\mathrm{BE}_{\mathrm{IL}}$, Fig. 6.

Fig. 7 shows binding energies of the interaction between the ionic liquid and different nanosheets $\left(\mathrm{BE}_{\mathrm{IL}-\mathrm{S}}\right)$. At the PBE/DZP level $\mathrm{BE}_{\mathrm{IL}-\mathrm{S}}$ follows as: graphene $\simeq$ boron-nitride $<$ germanene $<$ silicene. The adsorption of $[\mathrm{CH}][\mathrm{BE}]$ on top of graphene and boron-nitride yields $\mathrm{BE}_{\mathrm{IL}-\mathrm{S}} \sim 12.24 \mathrm{kcal} \mathrm{mol}^{-1}$, while the values increase up to $19.41 \mathrm{kcal} \mathrm{mol}^{-1}$ and $34.49 \mathrm{kcal} \mathrm{mol}^{-1}$ for Ge and Si based sheets, respectively. As previously noted, interactions between choline benzoate and silicene lead to surface distortion, which allows a short intermolecular distance between the cation and the surface $\left(d_{8}\right.$, see Fig. 3$)$. However, this effect is not displayed in the IL-germanene system. At the PBE-D2/DZP level, computed $\mathrm{BE}_{\mathrm{IL}-\mathrm{S}}$ values follow a similar pattern, however both IL-silicene and IL-germanene systems yield similar 


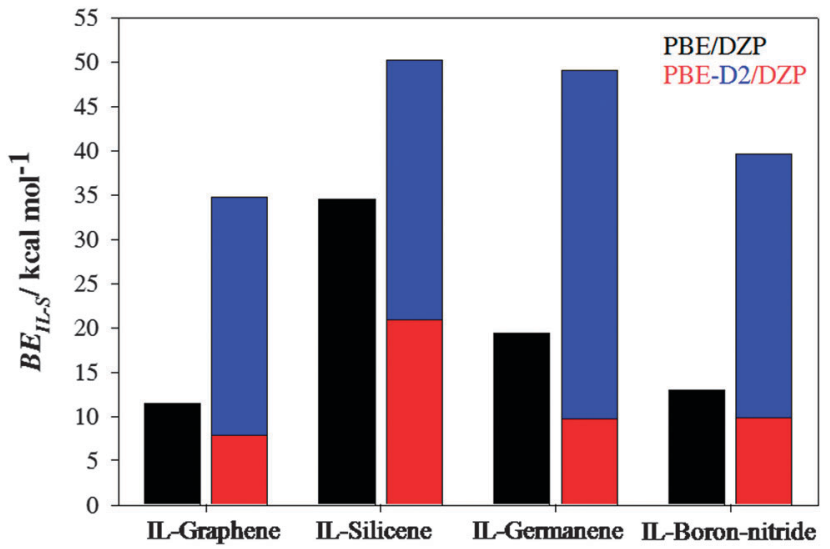

Fig. 7 Binding energies for the interaction between the IL and the nanosheets (BE $\mathrm{IL}_{\mathrm{LS}}$ ) at PBE/DZP (black) and PBE-D2/DZP (red + blue) theoretical levels, wherein blue bar stands for $\mathrm{BE}_{\text {dis }}$ contributions.

$\mathrm{BE}_{\mathrm{IL}-\mathrm{S}}\left(\simeq 49.65 \mathrm{kcal} \mathrm{mol}^{-1}\right)$. Fig. 7 also draws the contribution from $\mathrm{BE}_{\mathrm{dis}, \mathrm{IL}-\mathrm{S}}$ to the total $\mathrm{BE}_{\mathrm{IL}-\mathrm{S}}$ estimated using the PBE-D2 functional. Note that $\mathrm{BE}_{\mathrm{dis}, \mathrm{IL}-\mathrm{S}} \simeq 28.67 \mathrm{kcal} \mathrm{mol}^{-1}$, except for IL-germanene systems, whose $\mathrm{BE}_{\mathrm{dis}, \mathrm{IL}-\mathrm{S}}=39.43 \mathrm{kcal} \mathrm{mol}^{-1}$. As seen in Tables 2-5, most intermolecular IL- germanene distances are, in general, shorter than those obtained for the remaining systems, which would lead to an enhancement of the dispersion contribution. These high $\mathrm{BE}_{\mathrm{dis}, \mathrm{IL}-\mathrm{s}}$ values prove that dispersion interactions are the main driving forces between the selected IL and the surfaces. Those results agree with iso-surface features described previously. In general, the highest values obtained for IL-silicene and IL-germanene systems would be due to their buckled honeycomb structure, which allow a higher chemical reactivity than graphene, leading to a much stronger adsorption of atoms and molecules. ${ }^{1,19,20}$

Previously reported studies estimated that $\mathrm{BE}_{\mathrm{IL}-\mathrm{S}}$ for different ionic liquid on top of graphene ${ }^{8}$ or boron-nitride ${ }^{29}$ sheets were roughly $63.00 \mathrm{kcal} \mathrm{mol}^{-1}$ (at the PBE-D2/DZP level) and $19.74 \mathrm{kcal} \mathrm{mol}^{-1}$ (PBE-D3/TZP), respectively. In these studies, nanosheets mainly tends to interact with the cation. However in this work, the larger contribution to the $\mathrm{BE}_{\mathrm{IL}-\mathrm{S}}$ comes from anion-sheet interactions.

\subsection{Electronic structure}

Fig. 8 shows the density of states (DOS) for $[\mathrm{CH}][\mathrm{BE}]$ and $[\mathrm{CH}][\mathrm{BE}]-$ nanosheet systems at $\mathrm{PBE} / \mathrm{DZP}$ (although similar results can be drawn using the PBE-D2 functional). DOS for pristine nanosheets, partial density of states corresponding to the $[\mathrm{CH}][\mathrm{BE}]$ ionic liquids, as well as the contributions from both ions are also reported in Fig. 8. There are several studies dealing with the electronic structure of pristine nanosheets, ${ }^{1,18,19,40,54}$ and the results reported in Fig. 8 are in agreement with the available data, which show zero gap semiconductor characteristics for all cases, except for boron-nitride nanosheets which show the insulator character (wide gap). The adsorption of choline benzoate ionic liquid on graphene, silicene or germanene does not have a dramatic effect on the nanosheet electronic structure, Fig. 8, with only a slight increase the energies of both valence/conductivity
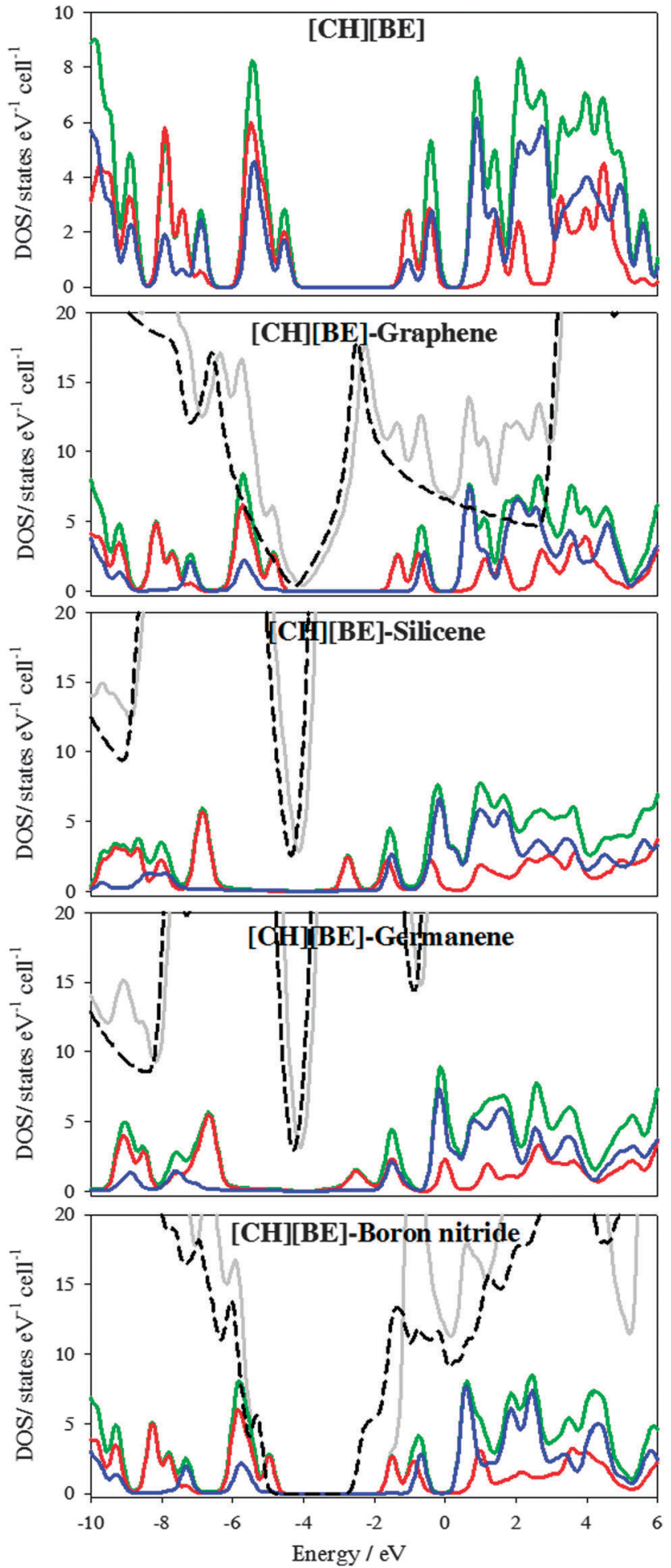

Fig. 8 Density of States calculated at the PBE/DZP theoretical level for pristine nanosheets (dotted line), [CH][BE] and [CH][BE]-nanosheets systems calculated at PBE/DZP levels (grey). Partial density of states (PDOS) for the ionic pair (green) as well as benzoate (red) and choline (blue) ions are also shown. Similar contours are obtained for the PBE-D2 functional.

bands $(\sim 0.20 \mathrm{eV})$. For IL on silicene and germanene, the highest/ lowest occupied/unoccupied orbitals corresponding to the ionic liquid (i.e. the HOMO and the LUMO over the ionic liquid motif) 


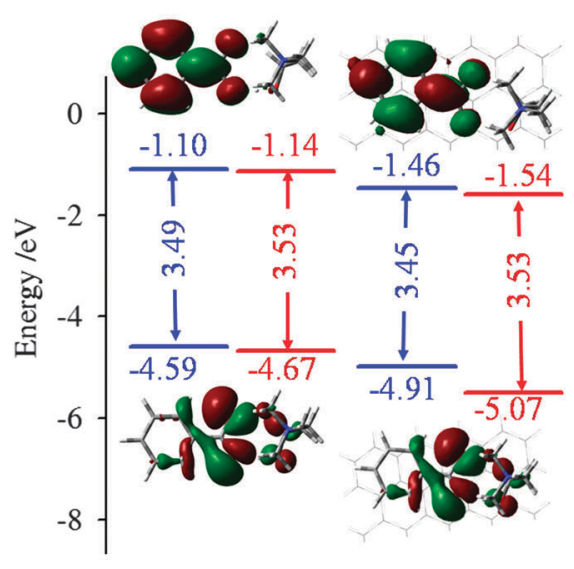

$[\mathrm{CH}][\mathrm{BE}]$

IL-Graphene

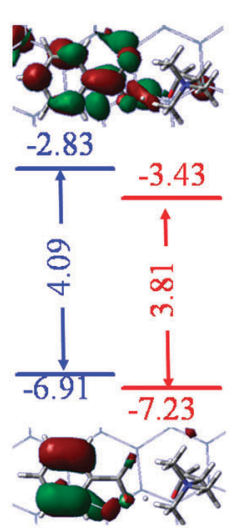

IL-Silicene

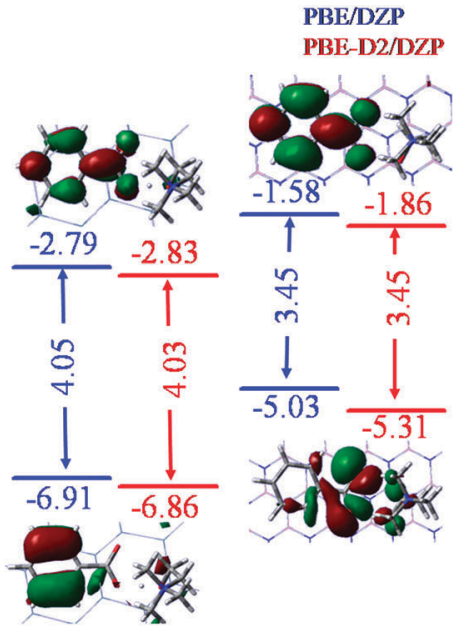

IL-Germanene IL-Boron-nitride

Fig. 9 Molecular orbital contours corresponding to the highest/lowest occupied/unoccupied molecular orbitals (HOMO-LUMO) located over the $[\mathrm{CH}][\mathrm{BE}]$ ionic liquid calculated at PBE/DZP levels. Similar contours are obtained for the PBE-D2 functional. HOMO and LUMO energies, along HOMOLUMO energy differences, are also indicated.

are far from the valence and conduction bands of the ILnanosheet systems. The same features are also noted for the LUMO over IL for IL-graphene systems, while the HOMO is closer in energy to the valence band. Therefore, IL adsorption does not change its zero band gap character. With regards to the boron-nitride nanosheet, the presence of IL leads to a decrease of its band gap, from $3.93 \mathrm{eV}$ up to $3.45 \mathrm{eV}$ (at the PBE/DZP level). After the adsorption process, the highest occupied level is due to the $\mathrm{IL}$, which is higher in energy that the boron-nitride valence band. In addition, interaction with the IL leads to conductivity band destabilization, which yields energies near to the LUMO.

From PDOS plotted in Fig. 8 information about cation and anion contributions to their corresponding HOMOs and LUMOs is inferred. The same information is easily obtained from the molecular orbital contours shown in Fig. 9. The LUMO of the isolated ionic liquid is mainly localized over the benzoate anion. The same behavior is also found for $[\mathrm{CH}][\mathrm{BE}]$ on top of the selected nanosheets. The adsorption on graphene/boron-nitride nanosheets leads to a decrease in the LUMO energy of $0.36 \mathrm{eV} /$ $0.48 \mathrm{eV}$, while the adsorption on silicene/germanene leads to a decrease in the LUMO energy of $\simeq 1.71 \mathrm{eV}$ (at the PBE/DZP level). Similar patterns are obtained for the HOMO energies, i.e. the adsorption on graphene, boron-nitride or silicene/germanene leads to a decrease in a HOMO energies of $0.32 \mathrm{eV}, 0.44 \mathrm{eV}$ or $2.32 \mathrm{eV}$. Hence, isolated ionic liquid as well as ionic liquid on top of graphene or boron-nitride surfaces yield a HOMO-LUMO difference of around $3.46 \mathrm{eV}$; while the larger HOMO energy change for choline benzoate on top of silicene or germanene provides a HOMO-LUMO difference of $\simeq 4.07 \mathrm{eV}$ (at PBE/DZP level). However, the contribution from each ion to the HOMO varies as a nanosheet function. The HOMO is located over the choline motif, although it also shows a very important contribution from the benzoate anion, for isolated $[\mathrm{CH}][\mathrm{BE}]$ and choline benzoate on top of the graphene/boron-nitride sheet. For these systems, interionic CT is $\simeq 0.32 e^{-} / 0.51 e^{-}$ (according ChelpG/Hirshfeld models), while CT from the benzoate to the sheet is close to zero (for both ChelpG/Hirshfeld schemes) at the PBE/DZP theoretical level. For the ionic liquid on top of silicene or germanene sheets, interionic CT is $\simeq 0.28 e^{-}$or $0.19 e^{-}$, respectively, according to Hirshfeld populations. Both IL-silicene and IL-germanene systems yield an important CT from the ionic liquid (concretely from the anion) to the sheet, equal to $0.54 e^{-}$and $0.68 e^{-}$, respectively. These high CT values lead to the HOMO being mainly located on the benzoate anion. In addition, these HOMO contours also show some similarities with the HOMO-1 orbital for the pristine ionic liquid. ILnanosheet CTs according to the ChelpG model are close to zero. There should be a relationship between the IL-sheet CT transfer according to the Hirshfeld method and the HOMO energy: the greatest/smallest CT is obtained for those nanosheets which are able to provide the lowest/highest HOMO (from the ionic liquid) energy. Therefore, the Hirshfeld model could be more appropriate to study the adsorption of IL on top of nanosheets.

\section{Conclusions}

The adsorption of choline benzoate ionic liquid on the surface of 2D nanosheets, graphene, silicene, germanene and boronnitride was studied using the DFT approach. The interaction mechanisms, binding energies and electronic structures were calculated using the PBE functional as well as PBE plus dispersion corrections (PBE-D2). The latter provided useful information on the contributions from dispersion forces to the interaction between both ions (which is mainly due to Coulombic forces) as well as the interaction between the IL and the nanosheet, which is mainly due to dispersion forces. Charge transfer from the anion to the surface also plays an important role. There is also a relationship between interionic and ionic liquid-nanosheet interactions. Therefore, the larger interionic binding energies 
are obtained for choline benzoate on top of carbon and boron nitride based sheets, which provides the lowest IL-sheet interaction energies. The analysis results showed that the considered sheets interact with the anion through $\pi$-stacking, although there is also a remarkable charge transfer in IL-silicene and IL-germanene. The electronic structure analysis exposed that the nanosheet electronic structure is not affected during the IL adsorption process. However, HOMO and LUMO molecular orbitals located on the IL suffer changes in their energies upon adsorption. Thus, the largest changes are noted for choline benzoate on top of silicene and germanene sheets. For both systems, there is also a larger charge transfer from the $\mathrm{IL}$ to the sheet, which leads to changes in the molecular fragment contributions to the HOMO contours. In this work, ChelpG and Hirshfeld models have been used to compute charge transfer processes, with results showing that only the Hirshfeld method would provide coherent values for IL-sheet charge transfer processes and their relationship with binding energies or with the molecular orbital shapes. In short, IL-graphene and IL-BN would provide similar features (such as interaction mechanism, CT, binding energies or electronic structure), while IL-silicene and IL-germanene would bring greater changes on assessed properties.

The reported results provide useful information on the ionic liquid adsorption on different nanosheets and their interaction mechanisms. They could also provide insights for the tuning of ionic liquid properties through the adsorption on the adequate nanosheet.

\section{Acknowledgements}

This work was made possible by Ministerio de Economía y Competitividad (Spain, project CTQ2013-40476-R) and Junta de Castilla y León (Spain, project BU324U14). Gregorio García acknowledges the funding by Junta de Castilla y León, cofounded by European Social Fund, for a postdoctoral contract. We also acknowledge The Foundation of Supercomputing Center of Castile and León (FCSCL, Spain), Computing and Advanced Technologies Foundation of Extremadura (CénitS, LUSITANIA Supercomputer, Spain), and Consortium of Scientific and Academic Services of Cataluña (CSUC, Spain) for providing supercomputing facilities. We also thank Dr S. Cahangirov and S. Ciraci (Bilkent Unversity) for providing us the structures of pristine graphene, silicene and germanene nanosheets. The statements made herein are solely the responsibility of the authors.

\section{References}

1 M. Xu, T. Liang, M. Shi and H. Chen, Chem. Rev., 2013, 113, 3766-3798.

2 H. Y. Mao, S. Laurent, S. Chen, W. O. Akhavan, M. Imani, A. A. Ashkarran and M. Mahmoudi, Chem. Rev., 2013, 113, 3407-3424.

3 K. S. Novoselov, A. K. Geim, S. V. Morozov, D. Jiang, Y. Zhang, S. V. Dubonos, I. V. Grigorieva and A. A. Firsov, Science, 2004, 306, 666-669.
4 J. K. Wassei and R. B. Kaner, Acc. Chem. Res., 2013, 46, 2244-2253.

5 X. Hu and Q. Zhou, Chem. Rev., 2013, 113, 3815-3835.

6 V. Georgakilas, M. Otyepka, A. B. Bourlinos, V. Chandra, N. Kim, K. C. Kemp, P. Hobza, R. Zboril and K. S. Kim, Chem. Rev., 2012, 112, 6156-6214.

7 J. Björk, F. Hanke, C. A. Palma, P. Samori, M. Cecchini and M. Persson, J. Phys. Chem. Lett., 2010, 1, 3407-3412.

8 C. Herrera, R. Alcalde, M. Atilhan and S. Aparicio, J. Phys. Chem. C, 2014, 118, 9741-9757.

9 M. Sha, M. Wu, G. Y. Liu, Z. Tang and H. Fang, J. Phys. Chem. C, 2009, 113, 4618-4622; T. Fukushima and T. Aida, Chemistry, 2007, 13, 5048-5058; Y. Shim, Y. Jung and H. J. Kim, J. Phys. Chem. C, 2011, 115, 23574-23583.

10 S. Aparicio and M. Atilhan, J. Phys. Chem. C, 2012, 116, 12055-12065.

11 A. S. Pensado, F. Malberg, M. F. C. Gomes, A. A. H. Padua, J. Fernandez and B. Kirchner, RSC Adv., 2014, 4, 18017-18024.

12 M. Tunckol, J. Durand and P. Serp, Carbon, 2012, 50, 4303-4334.

13 S. Aparicio, M. Atilhan and F. Karadas, F., Ind. Eng. Chem. Res., 2010, 49, 9580-9595; J. S. Wilkes, Green Chem., 2002, 4, 73-80; J. Earle-Martyn and R. Seddon-Kenneth, Ionic Liquids: Green Solvents for the Future, Clean Solvents, American Chemical Society, 2002.

14 N. V. Plechkova and K. R. Seddon, Chem. Soc. Rev., 2008, 37, 123-150; Z. Lei, C. Dai and B. Chen, Chem. Rev., 2013, 114, 1289-1326.

15 H. Zhou, M. Rouha, G. Feng, S. S. Lee, H. Docherty, P. Fenter, P. T. Cummings, P. F. Fulvio, S. Dai, J. McDonough, V. Presser and Y. Gogotsi, ACS Nano, 2012, 6, 9818-9827; Q. Dou, M. L. Sha, H. Y. Fu and g. Z. Wu, J. Phys.: Condens. Matter, 2011, 23, 175001.

16 M. H. Ghatee and F. Moosavi, J. Phys. Chem. C, 2011, 115, 5626-5636; T. Carstens, R. Gustus, O. Höfft, N. Borisenko, F. Endres, H. Li, R. J. Wood, A. J. Page and R. Atkin, J. Phys. Chem. C, 2014, 118, 10833-10843; A. J. Page, A. Elbourne, R. Stefanovic, M. A. Addicoat, G. G. Warr, G. G. Voitchovsky and K. R. Atkin, Nanoscale, 2014, 6, 8100-8106; M. Vijayakumar, B. Schwenzer, V. Shutthanandan, J. Hu, J. Liu and I. A Aksay, Nano Energy, 2014, 3, 152-158; S. A. Kislenko, I. S. Samoylov and R. H. Amirov, Phys. Chem. Chem. Phys., 2009, 11, 5584-5590.

17 G. Kamath and G. A. Baker, Phys. Chem. Chem. Phys., 2012, 14, 7929-7933; D. Wagle, G. Kamath and G. A. Baker, J. Phys. Chem. C, 2013, 117, 4521-4532; S. Ravula, S. N. Baker, G. Kamath and G. A. Baker, Nanoscale, 2015, 7, 4338-4353. 18 D. Jose and A. Datta, Acc. Chem. Res., 2013, 47, 593-602.

19 A. Kara, H. Enriquez, A. P. Seitsonen, L. C. Lew Yan Voon, S. Vizzini, B. Aufray and H. Oughaddou, Surf. Sci. Rep., 2012, 67, 1-18.

20 W. Xia, W. Hu, Z. Li and J. Yang, Phys. Chem. Chem. Phys., 2014, 16, 22495-22498.

21 S. Wang, Phys. Chem. Chem. Phys., 2011, 13, 11929-11938.

22 Y. Tian, X. F. Pan, Y. J. Liu and J. X. Zhao, Appl. Surf. Sci., 2014, 295, 137-143. 
23 N. J. Roome and J. D. Carey, ACS Appl. Mater. Interfaces, 2014, 6, 7743-7750; N. Gao, W. T. Zheng and Q. Jiang, Phys. Chem. Chem. Phys., 2012, 14, 257-261; R. Wang, X. Pi, Z. Ni, Y. Liu and D. Yang, RSC Adv., 2015, 5, 33831-33837; B. Huang, H. J. Xiang and S.-H. Wei, Phys. Rev. Lett., 2013, 111, 145502.

24 T. H. Osborn, A. A. Farajian, O. V. Pupysheva, R. S. Aga and L. C. Lew Yan Voon, Chem. Phys. Lett., 2011, 511, 101-105.

25 W. Hu, N. Xia, X. Wu, Z. Li and J. Yang, Phys. Chem. Chem. Phys., 2014, 16, 6957-6962; T. P. Kaloni, G. Schreckenbach and M. S. Freund, J. Phys. Chem. C, 2014, 118, 23361-23367.

26 N. Ding, N. X. Chen, C.-M. L. Wu and H. Li, Phys. Chem. Chem. Phys., 2013, 15, 10767-10776.

27 H. Liu and C. H. Turner, Phys. Chem. Chem. Phys., 2014, 16, 22853-22860.

28 S. L. Tang, Y. J. Liu, H. X. Wang, J. X. Zhao, Q. H. Cai and X. Z. Wang, Diamond Relat. Mater., 2014, 44, 54-61.

29 M. Shakourian-Fard, G. Kamath and Z. Jamshidi, J. Phys. Chem. C, 2014, 118, 26003-26016.

30 G. Kamath and G. A. Baker, RSC Adv., 2013, 3, 8197-8202.

31 D. J. Couling, R. J. Bernot, K. M. Docherty, J. K. Dixon and E. J. Maginn, Green Chem., 2006, 8, 82-90.

32 M. Petkovic, J. L. Ferguson, H. Q. N. Gunaratne, R. Ferreira, M. C. Leitao, K. R. Seddon, L. P. N. Rebelo and C. S. Pereira, Green Chem., 2010, 12, 643-649.

33 Y. Yu, X. Lu, Q. Zhou, K. Dong, H. Yao and S. Zhang, Chemistry, 2008, 14, 11174-11182.

34 J. P. Perdew, K. Burke and M. Ernzerhof, Phys. Rev. Lett., 1996, 77, 3865.

35 J. M. Soler, E. Artacho, E. Gale, J. D. A. García, J. Junquera, P. Ordejón and D. Sánchez-Portal, J. Phys.: Condens. Matter, 2002, 14, 2745.

36 N. Troullier and J. L. Martins, Phys. Rev. B: Condens. Matter Mater. Phys., 1991, 43, 1993-2006.

37 H. J. Monkhorst and J. D. Pack, Phys. Rev. B: Solid State, 1976, 13, 5188-5192.

38 J. G. Hernández, E. Anota, M. R. de la Cruz, M. Melchor and G. Cocoletzi, J. Mol. Model., 2012, 18, 3857-3866; R. R. Q. Freitas,
R. R. Q. Rivelino, R. F. D. B. Mota and C. M. C. de Castilho, J. Phys. Chem. A, 2011, 115, 12348-12356; T. Hu and I. C. Gerber, J. Phys. Chem. C, 2013, 117, 2411-2420.

39 C. Lechner and A. F. Sax, J. Phys. Chem. C, 2014, 118, 20970-20981.

40 J. W. Feng, Y. J. Liu, H. X. Wang, J. X. Zhao, Q. H. Cai and X. Z. Wang, Comput. Mater. Sci., 2014, 87, 218-226.

41 Y. Ma, Y. Dai, C. Niu and B. Huang, J. Mater. Chem., 2012, 22, 12587-12591.

42 A. J. Cohen, P. Mori-Sánchez and W. Yang, Chem. Rev., 2012, 112, 289-320.

43 Q. Sun, Z. Li, D. J. Searles, Y. Chen, G. Lu and A. Du, J. Am. Chem. Soc., 2013, 135, 8246-8253.

44 S. Grimme, J. Comput. Chem., 2006, 27, 1787-1799.

45 F. L. Hirshfeld, Theor. Chim. Acta, 1977, 44, 129-138.

46 C. M. Breneman and K. B. Wiberg, J. Comput. Chem., 1990, 11, 361-373.

47 S. Aparicio and M. Atilhan, Chem. Phys., 2012, 418, 118-125; S. Aparicio and M. Atilhan, Energy Fuels, 2013, 27, 2515-2527; V. Sanz, R. Alcalde, M. Atilhan and S. Aparicio, J. Mol. Model., 2014, 20, 1-14; S. Aparicio and M. Atilhan, Energy Fuels, 2010, 24, 4989-5001.

48 S. Aparicio and M. Atilhan, J. Phys. Chem. B, 2012, 116, 9171-9185.

49 R. F. W Bader, Atoms in Molecules: a Quantum Theory, Oxford, 1990.

50 E. R. Johnson, S. Keinan, P. Mori-Sánchez, J. ContrerasGarcía, A. J. Cohen and W. Yang, J. Am. Chem. Soc., 2010, 132, 6498-6506.

51 T. Lu and F. Chen, J. Comput. Chem., 2012, 33, 580-592.

52 A. Postnikov, http://www.home.uni-osnabrueck.de/apostnik/ download.html.

53 Y. Zhao and D. G. Truhlar, J. Chem. Theory Comput., 2006, 3, 289-300.

54 S. Cahangirov and S. Ciraci, Phys. Rev. B: Condens. Matter Mater. Phys., 2011, 83, 165448; S. Cahangirov, M. Topsakal, E. Aktürk, H. Şahin and S. Ciraci, Phys. Rev. Lett., 2009, 102, 236804. 